\title{
Coherent interface strengthening of ultrahigh pressure heat-treated Mg-Li-Y alloys.
}

ZHANG, S., SUN, Y., WU, R., WANG, X., CHEN, X.-B., FERNANDEZ, C., and PENG, $Q$. 


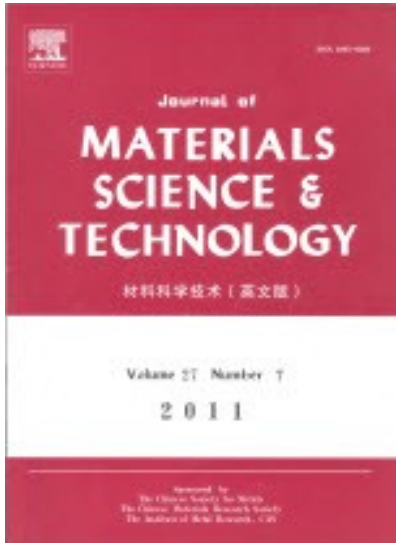

\section{Coherent Interface Strengthening of Ultrahigh Pressure Heat-Treated Mg-Li-Y Alloys}

\begin{tabular}{|c|c|}
\hline Journal: & Journal of Materials Science \& Technology \\
\hline Manuscript ID & JMST-2019-3402.R1 \\
\hline Manuscript Type: & Letter \\
\hline $\begin{array}{r}\text { Date Submitted by the } \\
\text { Author: }\end{array}$ & 27-Dec-2019 \\
\hline Complete List of Authors: & $\begin{array}{l}\text { Zhang, Shun; Yanshan University, State Key Laboratory of Metastable } \\
\text { Materials Science and Technology; Harbin Engineering University, Key } \\
\text { Laboratory of Superlight Materials \& Surface Technology, Ministry of } \\
\text { Education } \\
\text { Sun, Yong; Yanshan University, State Key Laboratory of Metastable } \\
\text { Materials Science and Technology } \\
\text { Wu, Ruizhi; Harbin Engineering University, Key Laboratory of Superlight } \\
\text { Materials \& Surface Technology, Ministry of Education } \\
\text { Wang, Xiang; Harbin Engineering University, Key Laboratory of } \\
\text { Superlight Materials \& Surface Technology, Ministry of Education } \\
\text { Chen, Xiao-Bo; RMIT University, School of Engineering } \\
\text { Fernandez, Carlos; Robert Gordon University, School of Pharmacy and } \\
\text { life sciences } \\
\text { Peng, Qiuming; Yanshan University, State Key Laboratory of Metastable } \\
\text { Materials Science and Technology }\end{array}$ \\
\hline Keywords: & Mg alloys, Nano-twins, Stacking faults, Strengthening \\
\hline Speciality: & Magnesium Alloys \\
\hline
\end{tabular}

\section{SCHOLARONE" Manuscripts}





\title{
Letter
}

\section{Coherent Interface Strengthening of Ultrahigh Pressure Heat-Treated Mg-Li-Y Alloys}
Shun Zhang 1,2, Yong Sun ${ }^{1}$, Ruizhi Wu ${ }^{2}$, Xiang Wang *,2, Xiao-Bo Chen ${ }^{3}$, Carlos Fernandez ${ }^{4}$, Qiuming Peng *,1
${ }^{1}$ State Key Laboratory of Metastable Materials Science and Technology, Yanshan University, Qinhuangdao, 066004, China

${ }^{2}$ Key Laboratory of Superlight Materials \& Surface Technology, Ministry of Education, Harbin Engineering University, Harbin, 150001, China

${ }^{3}$ School of Engineering, RMIT University, Carlton 3053, VIC, Australia

${ }^{4}$ School of Pharmacy and life sciences, Robert Gordon University, Aberdeen, AB107GJ, UK

* Corresponding author. Prof. Qiuming Peng; Prof. Xiang Wang

E-mail address: pengqiuming@ysu.edu.cn (Qiuming Peng); wangxiang@hrbeu.edu.cn (Xiang Wang)

\begin{abstract}
Achieving good strength-ductility of $\mathrm{Mg}$ alloys has always been a crucial issue for the widespread applications of Mg-based structural materials. Herein, an unexpected double-stage strengthening phenomenon was discovered in Mg-8Li-1Y (wt.\%) alloys through high-pressure $(6 \mathrm{GPa})$ heat treatments over a range of $700-1300{ }^{\circ} \mathrm{C}$. Attractively, the yield strength values are improved remarkably without losing their ductility. The low-temperature strengthening mechanism is mainly driven by the formation of large-volume nanoscale contraction twins. In contrast, the high-temperature strengthening reason is ascribed to the presence of densely nano-sized stacking faults. Both coherent interfaces contribute effectively to high mechanical strength without any tradeoff in ductility.
\end{abstract}

Key words: Mg alloys; Nano-twins; Stacking faults; Strengthening 


\section{Introduction}

Mg alloys, the lightest structural metals, play an important role in energy saving and emission reduction [1]. However, due to the low strength and poor formability at room temperature, their engineering applications are still unsatisfied. A large number of approaches have been attempted to improve the strength of Mg alloys in the past decades [2]. Traditional strengthening strategies, such as grain refinement, precipitation and sub-grain boundaries through dislocation plugging, could introduce a large number of boundaries into $\mathrm{Mg}$ matrix, which can effectively prevent dislocation motion [2]. However, it is common to see the increment in mechanical strength at the expense of ductility through those incoherent interfaces, which is ascribed to the limited capacity to accommodate dislocations [3]. Hence, the feasible approach to attain high strength without scarification in plasticity remains a challenge for the wide applications of $\mathrm{Mg}$ alloys.

Differing from incoherent boundaries, coherent twin boundaries (TBs), can not only act as effective barriers to dislocation movement, but also provide huge space for dislocation storage. This strategy is applicable for FCC metals [4] whilst difficult for $\mathrm{HCP} \mathrm{Mg}$, owing to the intrinsic characteristics of twins in Mg alloys. In particular, the interfaces of [1012] tension twins (TTWs) are not stable. In addition, though the interfaces of [1011] contraction twins (CTWs) are more stable than those of TTWs, secondary TTWs are readily initiated in and occupy the entire internal domains of CTWs [5]. Therefore, it is difficult to strengthen Mg alloys by increasing volume fractions of TBs. More recently, our group introduced distinctive hierarchical $\{10 \overline{1} 1\}-\{1011\}$ double contraction nanotwins in $\mathrm{Mg}-8 \mathrm{Li}$ (all compositions given thereafter in wt.\%) binary alloy by means of an ultra-high pressure treatment (UHP) technique [6]. Such a unique structure elevates the yield strength of $\mathrm{Mg}-8 \mathrm{Li}$ alloys up from 50 to $249 \mathrm{MPa}$ while maintains its high ductility $(23.6 \%$ elongation rate). Subsequently, an improved contraction nanotwins-stacking faults structure was achieved by increasing Li concentration to $13 \mathrm{wt} . \%$, which further increases the specific strength of Mg-13Li alloy [7]. It is anticipated that coherent structure strengthening could be a feasible strategy to design high strength-ductile $\mathrm{Mg}$ alloys.

In the present work, we attempt to explore the effects of $\mathrm{Y}$ alloying element on interface strengthening of Mg-8Li alloys. It is well-known that large-sized rare earth elements (REs) are prone to segregate in TBs, which remarkably affects their structures and properties [8]. 
Experimental work was designed to provide evidence to elucidate two critical issues that dominate coherent interface strengthening in Mg alloys: i) is it technically feasible to yield coherent structure strengthening through REs alloying under milder conditions? and ii) which structure of contraction twins or stacking faults is more effective to improve mechanical properties?

\section{Experimental processes}

A Mg-8Li-0.5Zn-1Y (LZY801) ingot was prepared by vacuum induction melting and machined into samples with dimensions of $10 \mathrm{~mm}$ in diameter and $14 \mathrm{~mm}$ in length for following UHPed treatments. Samples were wrapped up with Ta foil and then inserted into a boron nitride crucible in a cubic-anvil large-volume press with six rams. Loading pressure of $6 \mathrm{GPa}$ was operated before heating up the system. After UHPed treatment holding at a defined temperature (over a range of $700-1300{ }^{\circ} \mathrm{C}$ ) for $30 \mathrm{~min}$, LZY801 specimens were quenched down to room temperature. The sample was then heated from room temperature to $200^{\circ} \mathrm{C}$ at a heating rate of $5^{\circ} \mathrm{C} / \mathrm{min}$ to release

residual stress. LZY801 samples which were subject to UHPed treatment under $6 \mathrm{GPa}$ at $900{ }^{\circ} \mathrm{C}$ for 30 min was denoted as LZY801-6GPa-900. The as-cast counterpart was introduced as a reference.

Microhardness was measured through a Vickers hardness tester (HV) with an applied load of 100 $\mathrm{g}$ and dwelling time of $15 \mathrm{~s}$. Mechanical tests were performed on Gleeble 3500 with a strain rate for tension and compression tests of $0.4 \mathrm{~mm} / \mathrm{min}$ and $1 \times 10^{-3} \mathrm{~s}^{-1}$, respectively. Length and width of the dog-bone shape tensile samples were 6 and $1.5 \mathrm{~mm}$, respectively. In comparison, compressive samples were accurately machined into dimensions of $5 \mathrm{~mm}$ in diameter and $10 \mathrm{~mm}$ in length. Microstructural investigations were performed using optical and transmission electron microscopy (TEM). TEM thin foils were prepared by Ar ion milling in a Fischione system operating at $5 \mathrm{keV}$. X-ray diffraction (XRD) was carried out on a Rigatu $2500 \mathrm{C}$ with $\mathrm{Cu}-\mathrm{K} \alpha$ radiation at a scanning rate of $4 \%$ min over a range of $20-80^{\circ}$.

\section{Results and discussion}

Figure 1a illustrates microhardness evolution of the UHPed samples as a function of processing temperature. The as-cast LZY801sample presents the lowest microhardness value of 48 
$\mathrm{HV}$, similar to that of previous as-cast $\mathrm{Mg}-8 \mathrm{Li}$ alloy [6]. It is noted that the samples are dramatically hardened $(75 \mathrm{HV})$ from the original as-cast state as a result of heat treatment $\left(700-1300{ }^{\circ} \mathrm{C}\right)$ at a constant high pressure $(6 \mathrm{GPa})$. Two distinct strengthening stages are evident between $700-900{ }^{\circ} \mathrm{C}$ and $1000-1200{ }^{\circ} \mathrm{C}$, respectively, followed by a sharp drop in microhardness from its peak point of $96 \mathrm{HV}\left(1200^{\circ} \mathrm{C}\right)$ to $54 \mathrm{HV}\left(1300^{\circ} \mathrm{C}\right)$.

In general, the melting point of a metal increases with externally applied pressure, such as the representative cases of Al-Zn alloys [9]. As such, two processes, i.e. ultra-high solid solution and ultra-high re-solidification, would occur during the UHPed heat treatments. Namely, the UHPed solid solution process occurs in a range of $700-1200{ }^{\circ} \mathrm{C}$, whilst the UHPed re-solidification is triggered above $1200{ }^{\circ} \mathrm{C}$, which is confirmed by both optical microstructure and XRD patterns (Figure 1b). Differing from $\alpha$ phase (Mg-rich) and $\beta$ phase (Li-rich), the eutectic $\mathrm{YZn}_{5}$ (hexagonal with space group $\mathrm{P} 6 / \mathrm{mmm}$ ) phase is reduced with increasing temperature, whilst the equal-axis grain structure well remains below $1200{ }^{\circ} \mathrm{C}$. Analogous to the atmospheric heat treatment, the UPed treatment mainly involves two typical processes. If the temperature is below melting point, the heat treatment is related to the UPed solid solution. Comparatively, when the temperature is over melting point, the heat treatment is ascribed to the UPed re-solidification. According to our previous results $[6,7]$, the melting point of $\mathrm{Mg}$ alloys will be increased to over $1200{ }^{\circ} \mathrm{C}$ under $6 \mathrm{GPa}$. As soon as the temperature is over melting point, the strengthening structure is eliminated, and the main strenthening role is merely related to solid-solution strengthening. Therefore, is demonstrated that the increment in microhardness of UHPed LZY801 is related to the UHPed solid solution treatment, while the decrease of microhardness is related to the ultra-high re-solidification.

Figure 1c displays the representative true stress-strain curves of the as-cast, the LZY801-6GPa-900 and the LZY801-6GPa-1200 specimens, which agrees with the results of microhardness measurements (Figure 1a). A low yield strength of $89 \mathrm{MPa}$ in the as-cast LZY801 alloy is largely increased up to $248 \mathrm{MPa}$ through heat treatment at $900{ }^{\circ} \mathrm{C}$ (LZY801-6GPa-900), and approaches the maximum value of $270 \mathrm{MPa}$ for the LZY801-6GPa-1200, three times of that of the as-cast one. It is noteworthy that such improvement in strength does not sacrifice elongation in a significant manner $(\sim 19 \%$, Figure 1c). Same results are confirmed by compressive tests, where samples are not fractured until it reaches the load limitation of compression tester (Figure 1d). In addition, the tensile-compression asymmetry (TCA)-the ratio of tensile yield strength 
$\left(\sigma_{\text {tys }}\right) /$ compressive yield strength $\left(\sigma_{\text {cys }}\right)$-is significantly minimized after UHPed treatment, and the TCA value is reduced from 1.25 (as-cast) to 1.02 (LZY801-6GPa-1200). In particular, tensile yield strength of all LZY801-6GPa alloys exceeds largely those of both Mg-Li binary alloys (100 \pm 50 $\mathrm{MPa}$ ) and majority of multiple Mg-Li-X alloy systems (X: other alloying elements), even after severe plastic deformation treatments, such as rolling [10-14], extrusion [14-17], equalchannel angular pressing [18, 19] and aging [20].

TEM characterization was employed to elucidate the mechanisms of the two-stage strengthening. Regarding the as-cast case (Figure 3a-c), both $\alpha$ and $\beta$ phases are evidently distributed in the matrix. Moreover, a number of particles with a size of $0.1 \mu \mathrm{m}$ in diameter are accumulated in grain boundaries. Corresponding energy dispersive spectroscopy (EDS) mapping (Figure 3b) confirms that the precipitates in the as-cast LZY801 sample are enriched in Y. Therefore, those particles are assigned to eutectic $\mathrm{YZn}_{5}$ phase, as indicated in the XRD pattern (Figure 1b). In the case of the LZY801-6GPa-900 (Figure 3d), a few nanoscale twins are embedded within the matrix, and some nanoscale particles are found. HRTEM micrograph (Figure 3e) exhibits that nano-twin boundaries (NTBs) are mainly composed of coherent structures, and the angle between (0001) twin and (0001) matrix is $\sim 123.4^{\circ}$, indicating the formation of CTWs [5]. The average twin thickness is about $80 \mathrm{~nm}$.

Figure 3f-g reveals that the dimension of the particles is $10-20 \mathrm{~nm}$. The spacing of (100) is about $0.453 \mathrm{~nm}$, which aligns will with the theoretical value $(0.432 \mathrm{~nm})$ of $\mathrm{YZn}_{5}$ [21]. Consequently, it is evident that the strengthening stage $\mathrm{I}$ is attributed to the presence of such a unique microstructure, i.e. co-presence of a large number of nano-coherent twins in matrix and a few residual nanoscale YZn ${ }_{5}$ phases. According to the empirical twin-strengthening mode [6],

theoretical yield strength with NTWs can be calculated by the following equation:

$$
\Delta \sigma_{\mathrm{y}}=n \mathrm{k}_{\mathrm{TB}} \lambda^{-1 / 2}
$$

Where $\lambda$ is $80 \mathrm{~nm} ; n$ is the volume fraction; and $\mathrm{k}_{\mathrm{TB}}$ is the Hall-Petch constant $\left(0.35 \mathrm{MPa} \mathrm{m}^{1 / 2}\right)$. Hence, the theoretical increment in yield strength of CTWs is $1.23 \mathrm{GPa}$.

In contrast, a large number of SFs with an average spacing of $18 \mathrm{~nm}$ exist in the matrix of LZY801-6GPa-1200 alloy without any twins or YZn particles (Figure 4a). The formation of SFs is also confirmed by the streaks in SAED (Figure $4 \mathbf{b}$ ). The atomic-scale image of SFs (Figure 4c) indicates that the SFs are assigned to growth fault ( $\mathrm{I}_{1}$-type), differing from the stacking sequence 
(...ABABABAB...) of an ideal $\mathrm{HCP} \mathrm{Mg}$ alloy. It is normally built by removing one A plane above one $\mathrm{B}$ plane, and then shearing the remaining planes above $\mathrm{B}$ plane by $1 / 3[1 \overline{100}]$, resulting in a ...ABACBCBCB... structure. According to previous SFs strengthening mechanisms, the theoretical increased yield strength can be calculated by the following equation [7]:

$$
\Delta \sigma_{\mathrm{y}}=m \mathrm{ksF}^{-1}
$$

Where $d$ is the average width of SFs $(18 \mathrm{~nm}) ; m$ is the volume fraction, and $\mathrm{k}_{\mathrm{SF}}$ is experimental constant (3780 MPa nm). Therefore, the theoretical increase in yield strength of SFs is $2.6 \mathrm{GPa}$.

\section{Conclusions}

The effect of Y alloying element on mechanical properties of the UHPed Mg-8Li alloys was explored. The results reveal that the addition alloying element can obviously optimize the UHPed conditions by reducing the operating temperature. Moreover, it demonstrates that both CTWs and SFs are achieved within one simple system by varying the UHPed conditions, and SFs are more effective to the enhancement in mechanical properties. The tradeoff between strength and ductility of $\mathrm{Mg}$ alloys can be mitigated through modifying nanoscale coherent interfaces.

\section{Acknowledgments}

We greatly acknowledge the financial support from NSFC (51771162 and 51971194) and Hebei province talent foundation (A201910002).

\section{References}

[1] X.J. Wang, D.K. Xu, R.Z. Wu, X.B. Chen, Q.M. Peng, L. Jin, Y.C. Xin, Z.Q. Zhang, Y. Liu, X.H. Chen, G. Chen, K.K. Deng, H.Y. Wang, J. Mater. Sci. Technol. 34 (2018) 245-247.

[2] T.M. Pollock, Science. 328 (2010) 986-987.

[3] W. Xu, N. Birbilis, G. Sha, Y. Wang, J.E. Daniels, Y. Xiao, M. Ferry, Nat. Mater. 14 (2015) 1229.

[4] X.Y. Li, Y.J. Wei, L. Lu, K. Lu, H.J. Gao, Nature. 464 (2010) 877-880.

[5] P. Cizek, M.R. Barnett, Scr. Mater. 59 (2008) 959-962.

[6] H. Fu, B.C. Ge, Y.C. Xin, R.Z. Wu, C. Fernandez, J.Y. Huang, Q.M. Peng, Nano. Lett. 17 (2017) 6117-6124. 
[7] Q.M. Peng, Y. Sun, B.C. Ge, H. Fu, Q. Zu, X.Z. Tang, J.Y. Huang, Acta. Mater. 169 (2019) 36-44.

[8] J.F. Nie, Y.M. Zhu, J.Z. Liu, X.Y. Fang, Science. 340 (2013) 957-960.

[9] B. Ahn, A.P. Zhilyaev, H.-J. Lee, M. Kawasaki, T.G. Langdon, Mat. Sci. Eng. A 635 (2015) 109-117.

[10] C.J. Shuai, W.J. Yang, Y.W. Yang, C.D. Gao, C.X. He, H. Pan, Int. J. Bioprint. 5 (2019) 49.

[11] G.Y. Sha, X.G. Sun, T. Liu, Y.H. Zhu, T. Yu, J. Mater. Sci. Technol. 27 (2011) 753-758.

[12] D.K. Xu, T.T. Zu, M. Yin, Y.B. Xu, E.H. Han, J. Alloys. Compd. 582 (2014) 161-166.

[13] T.L. Zhu, J.F. Sun, C.L. Cui, R.Z. Wu, S. Betsofen, Z. Leng, J.H. Zhang, M.L. Zhang, Mat. Sci. Eng. A 600 (2014) 1-7.

[14] M.L. Zhang, R.Z. Wu, T. Wang, J. Mater. Sci. 44 (2009) 1237-1240.

[15] C.Q. Li, D.K. Xu, B.J. Wang, L.Y. Sheng, R.Z. Wu, E.H. Han, J. Mater. Sci. Technol. 35 (2019) 2477-2484.

[16] Y. Tang, W.T. Jia, X. Liu, Q.C. Le, Y.L. Zhang, Mat. Sci. Eng. A 675 (2016) 55-64.

[17] Y. Yang, X.M. Xiong, J.F. Su, X.D. Peng, H.M. Wen, G.B. Wei, F.S. Pan, E.J. Lavernia, J. Alloys. Compd. 750 (2018) 696-705.

[18] M. Karami, R. Mahmudi, Mat. Sci. Eng. A 607 (2014) 512-520.

[19] R.Z. Wu, Z.K. Qu, M.L. Zhang, Rev. Adv. Mater. Sci. 24 (2010) 35-43.

[20] C.O. Muga, H. Guo, S.S. Xu, Z.W. Zhang, Mat. Sci. Eng. A 689 (2017) 195-202.

[21] M. Fornasini, J. Less-Common. Met. $25 \quad$ (1971) 329-332. 


\section{Figure captions}

Figure 1. Mechanical properties of UHPed LZY801 alloys as a function of processing temperature.

(a) Effects of UHP conditions on microhardness. (b) Optical microstructure and XRD patterns. (c) Tensile and (d) compression curves. The insets correspond to macro-size optical photographs.

Figure 2. Comparison in terms of mechanical properties between UHPed LZY801 alloys and a range of representative $\mathrm{Mg}-\mathrm{Li}-\mathrm{X}$ (alloying elements) alloys prepared by a variety of themal-mechanical treatments.

Figure 3. TEM characterization of the LYZ801-6GPa-900 alloy. (a) STEM micrographs of the as-cast LZY801 sample. (b) EDS mapping of Y in (a). (c) SAED pattern of $\alpha$-Mg. (d) TEM image of the LZY801-6GPa-900 observed along [11 20$]$ axis. A randomly particle in the matrix is marked by a red arrow. (e) Atomic-level image of coherent contraction twin boundary. (f) High magnification image of the particle in (d). (g) Local atomic-level magnified region as marked in (f).

Figure 4. Strengthening mechanisms in the LZY801-6GPa-1200 alloy. (a) TEM image with an inset corresponding to the spacing distribution SFs derived from statistical calculations of 50 random results. (b) Corresponding SAED patterns, where crystal planes and SFs (streaking pattern) are marked. (c) Atomic level image of SFs in (a). 

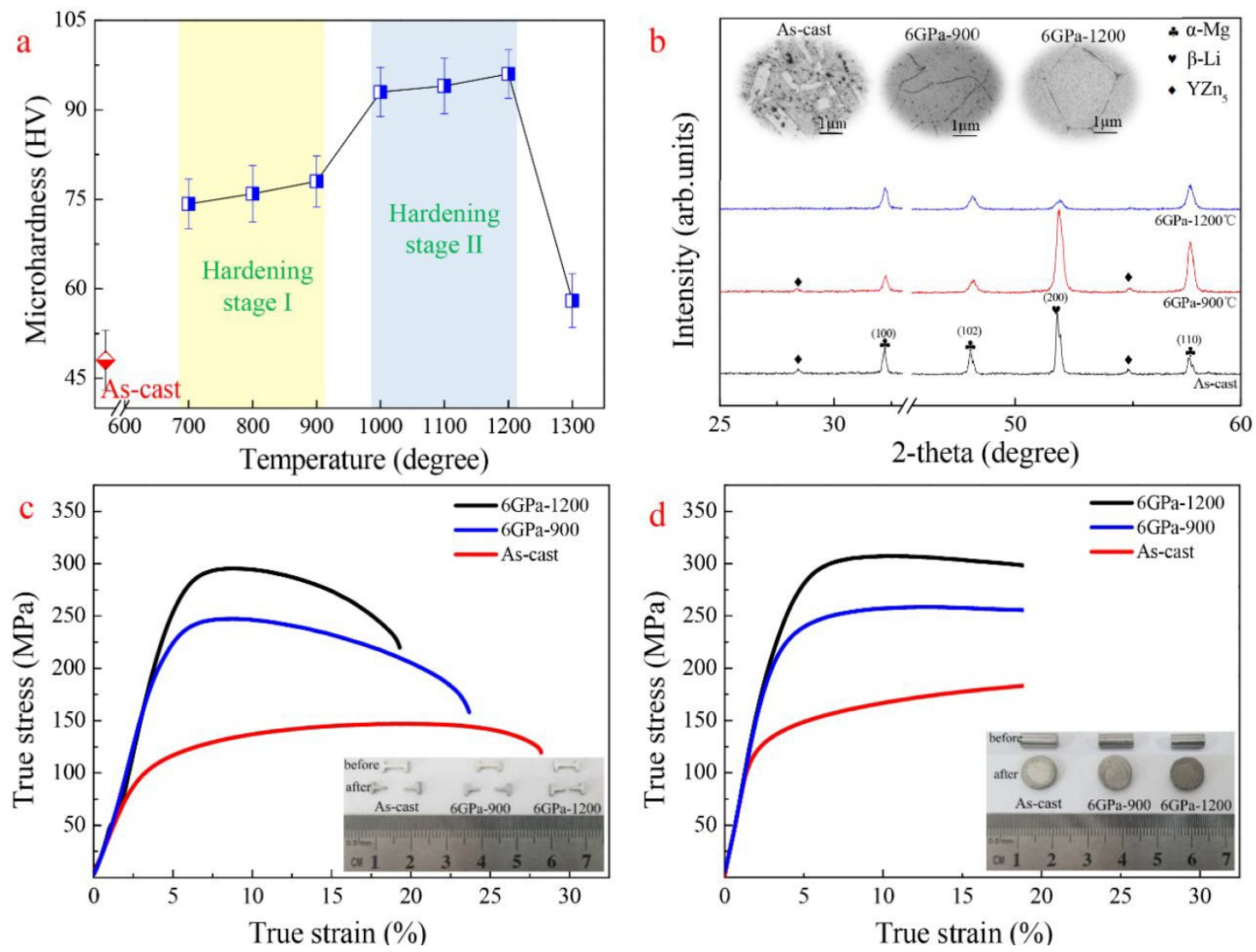

Figure 1. Mechanical properties of UHPed LZY801 alloys as a function of processing temperature. (a) Effects of UHP conditions on microhardness. (b) Optical microstructure and XRD patterns. (c) Tensile and (d) compression curves. The insets correspond to macro-size optical photographs. 


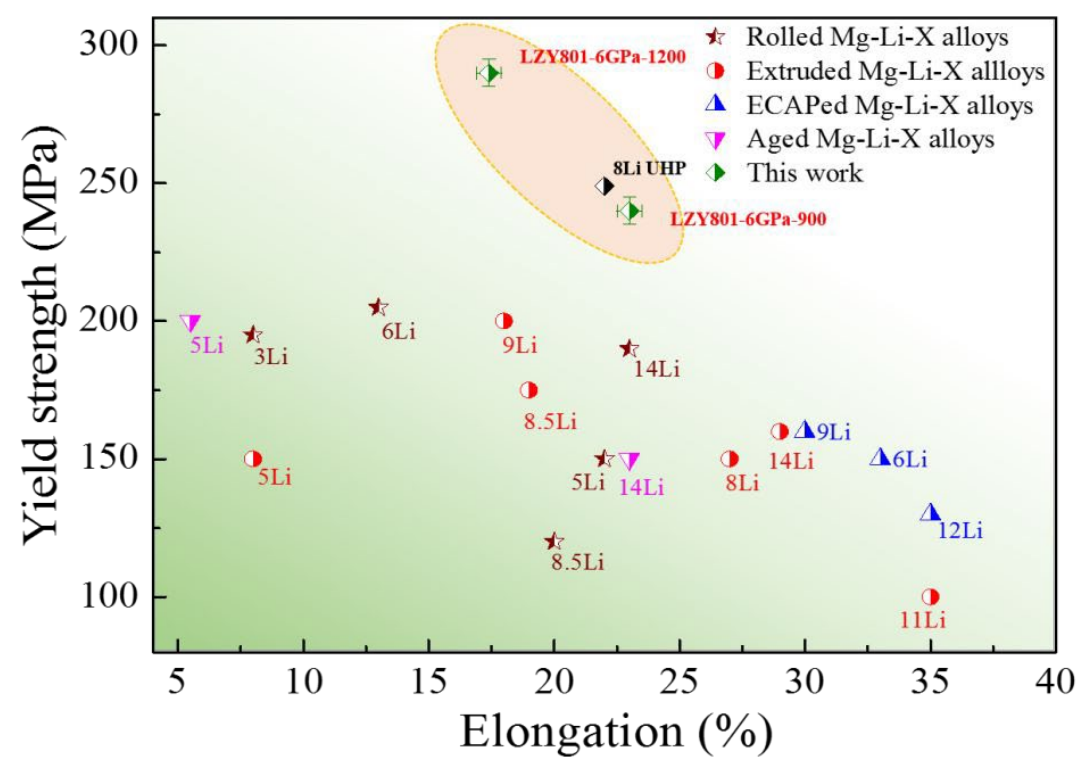

Figure 2. Comparison in terms of mechanical properties between UHPed LZY801 alloys and a range of representative $\mathrm{Mg}-\mathrm{Li}-\mathrm{X}$ (alloying elements) alloys prepared by a variety of themal-mechanical treatments. 

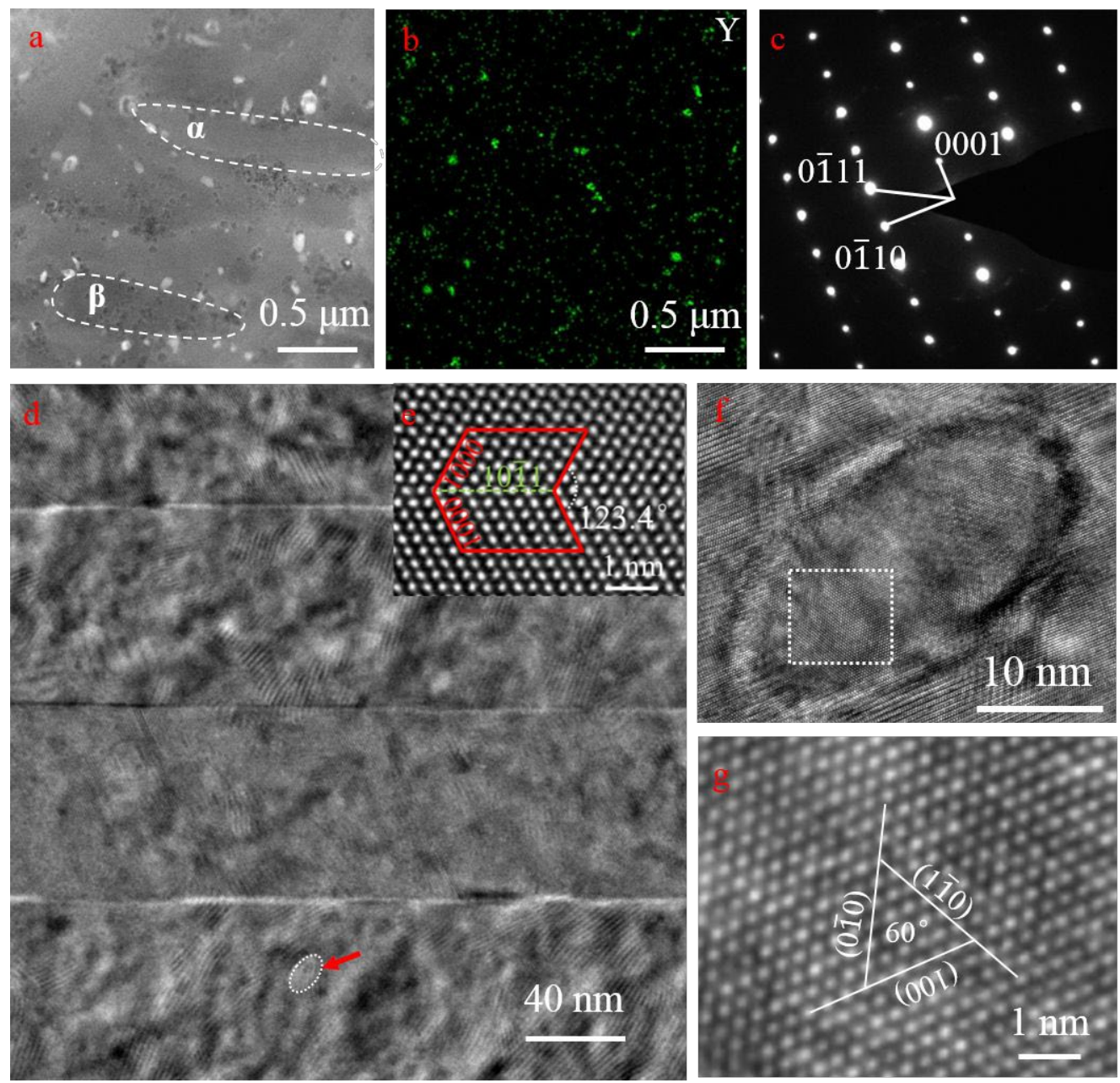

Figure 3. TEM characterization of the LYZ801-6GPa-900 alloy. (a) STEM micrographs of the as-cast LZY801 sample. (b) EDS mapping of Y in (a). (c) SAED pattern of $\alpha-\mathrm{Mg}$. (d) TEM image of the LZY801-6GPa-900 observed along [11후] axis. A randomly particle in the matrix is marked by a red arrow. (e) Atomic-level image of coherent contraction twin boundary. (f) High magnification image of the particle in (d). (g) Local atomic-level magnified region as marked in (f). 

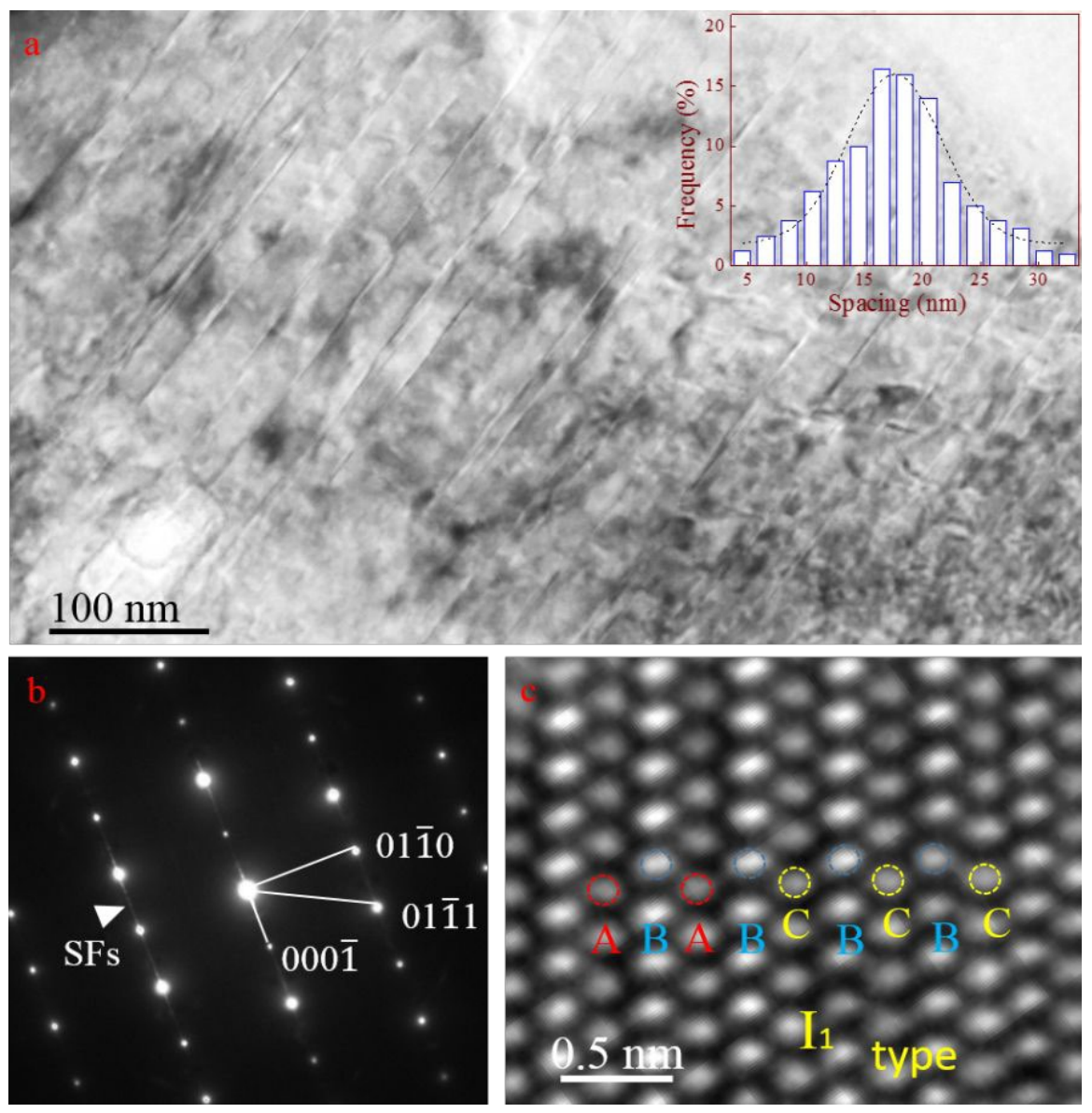

Figure 4. Strengthening mechanisms in the LZY801-6GPa-1200 alloy. (a) TEM image with an inset corresponding to the spacing distribution SFs derived from statistical calculations of 50 random results. (b) Corresponding SAED patterns, where crystal planes and SFs (streaking pattern) are marked. (c) Atomic level image of SFs in (a). 

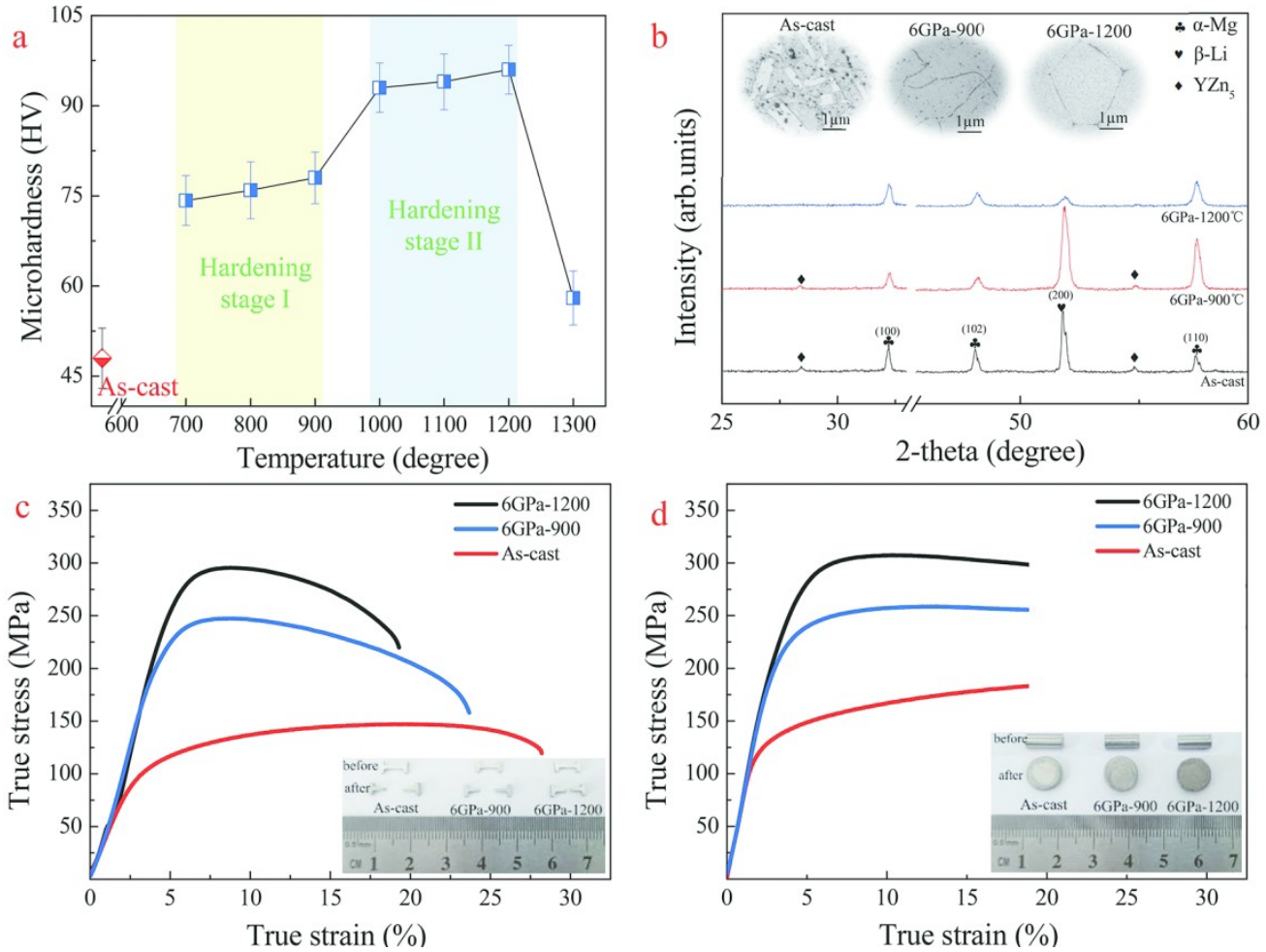

Figure 1. Mechanical properties of UHPed LZY801 alloys as a function of processing temperature. (a) Effects of UHP conditions on microhardness. (b) Optical microstructure and XRD patterns. (c) Tensile and (d) compression curves. The insets correspond to macro-size optical photographs. 


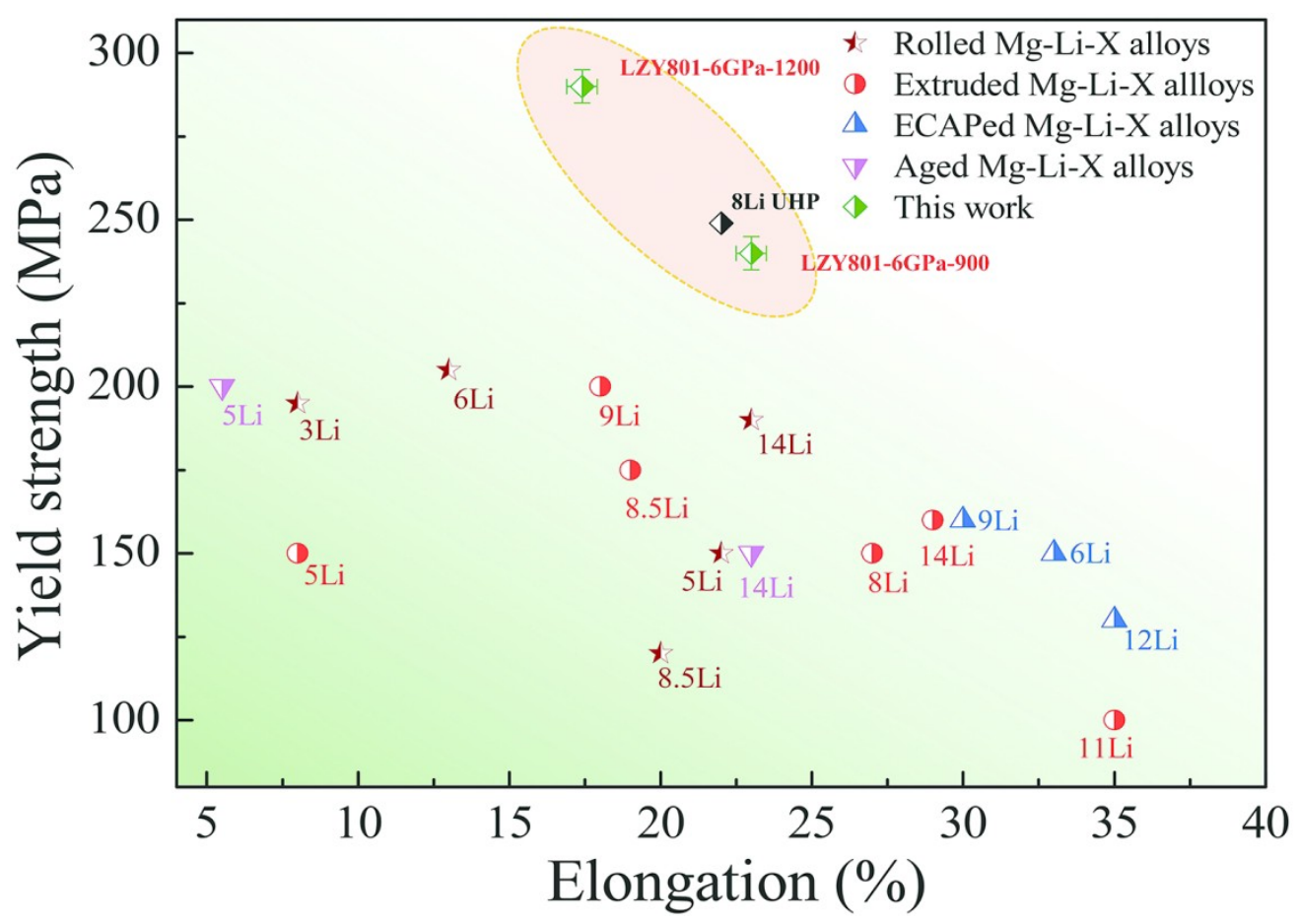

Figure 2. Comparison in terms of mechanical properties between UHPed LZY801 alloys and a range of representative Mg-Li-X (alloying elements) alloys prepared by a variety of themal-mechanical treatments. 

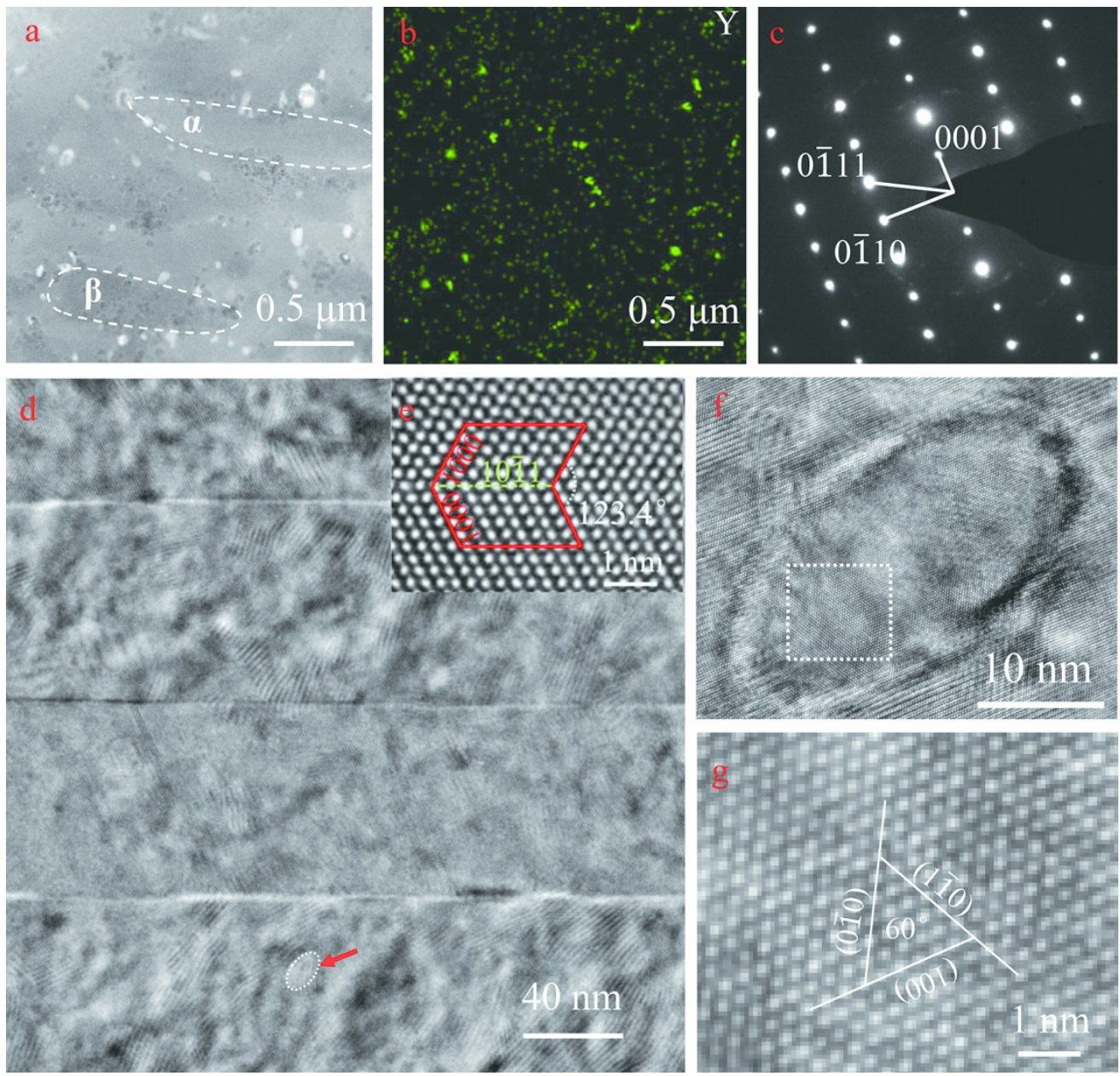

Figure 3. TEM characterization of the LYZ801-6GPa-900 alloy. (a) STEM micrographs of the as-cast LZY801 sample. (b) EDS mapping of $Y$ in (a). (c) SAED pattern of a-Mg. (d) TEM image of the LZY801-6GPa-900 observed along [11"2" 0] axis. A randomly particle in the matrix is marked by a red arrow. (e) Atomic-level image of coherent contraction twin boundary. (f) High magnification image of the particle in (d). (g) Local atomic-level magnified region as marked in (f). 


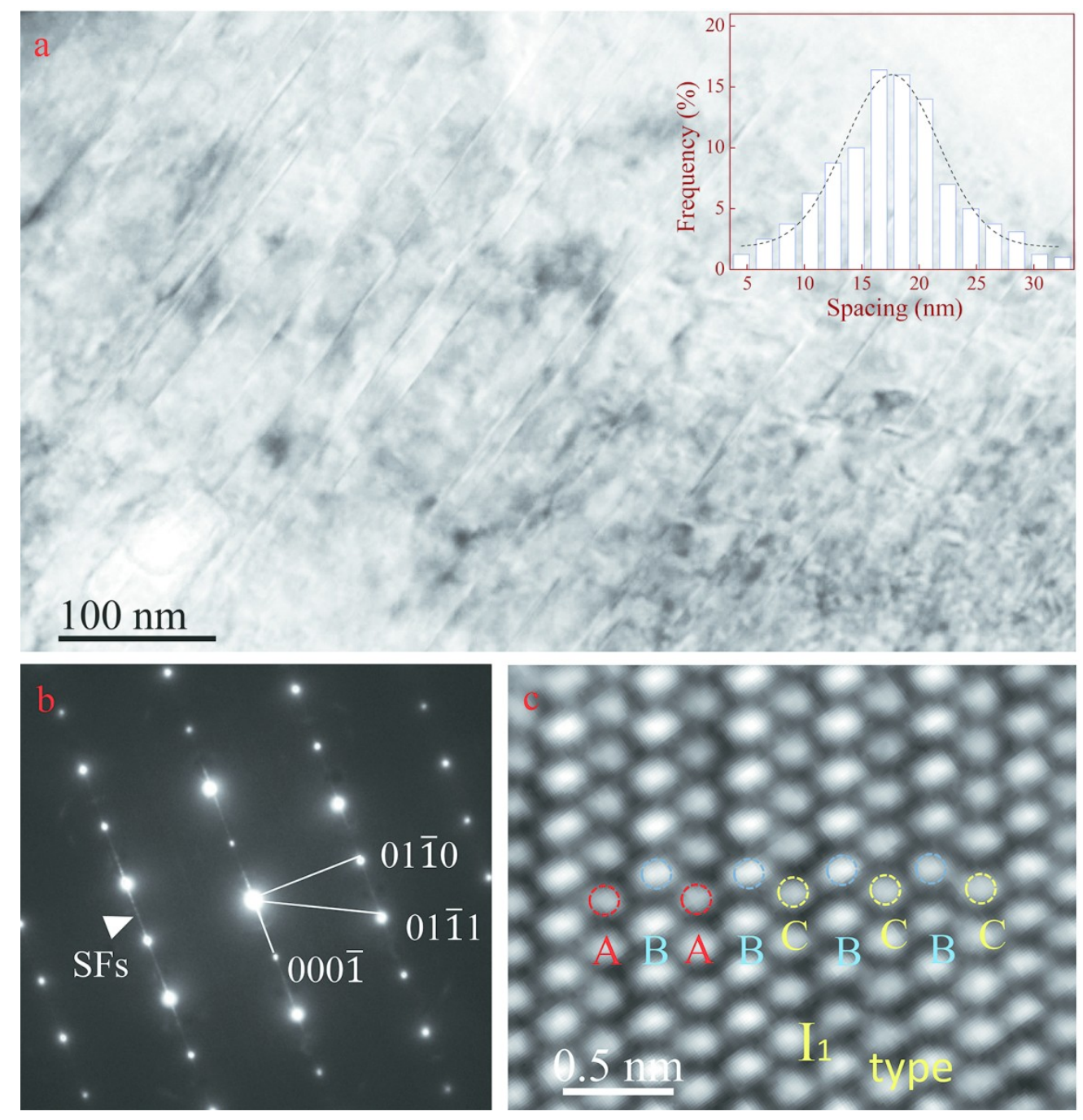

Figure 4. Strengthening mechanisms in the LZY801-6GPa-1200 alloy. (a) TEM image with an inset corresponding to the spacing distribution SFs derived from statistical calculations of 50 random results. (b) Corresponding SAED patterns, where crystal planes and SFs (streaking pattern) are marked. (c) Atomic level image of SFs in (a). 